\title{
Common causes of 'swelling' in the oral cavity
}

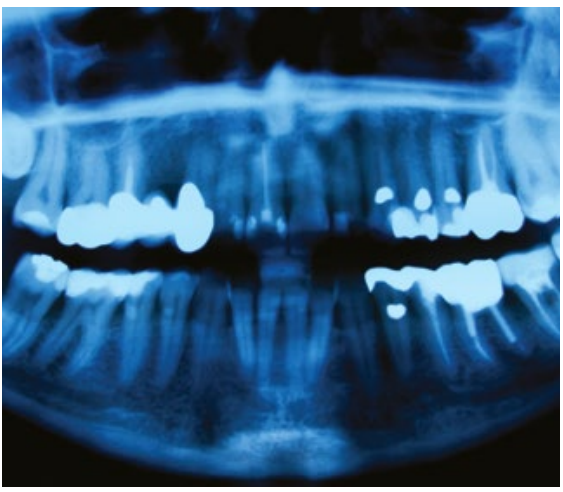

CPD

Timothy Wong, Tami Yap,

David Wiesenfeld

\section{Background}

Conditions that present as a 'swelling' in the oral cavity are relatively common, and patients may seek initial assessment from their general practitioners.

\section{Objective}

The aim of this article is to provide an overview of common causes of a 'swelling' in the oral cavity to help with formulating a differential diagnosis and stratifying the urgency of referral.

\section{Discussion}

Pathological conditions in the oral cavity (excluding mucosal presentations) may present as a swelling in the submucosa or jaws, symptoms related to teeth and/or gums or an incidental finding on imaging. In this review, the authors outline the most common submucosal or jaw swellings, organised according to their clinical presentations, and describe their typical appearance and management.
BROADLY SPEAKING, ORAL PATHOLOGY can present as a mucosal surface lesion (discussed in an accompanying article by these authors), ${ }^{1}$ swelling present at an oral subsite (lips/buccal mucosa, tongue, floor of mouth, palate and jaws) or symptoms related to teeth (pain, mobility). The last of these presentations has been excluded from this article as it is assumed patients with teeth-related symptoms are more likely to present to their dentists than their general practitioners.

The most commonly encountered swellings in the oral cavity are either submucosal in nature, or involve swelling of the underlying jaw (maxilla or mandible).

\section{The submucosal swelling}

\section{Mucocele}

A mucocele presents as a smooth, fluid-filled lump in areas with minor salivary glands that are commonly susceptible to oral trauma (eg lips, buccal mucosa; Figure 1). They occur when mucus/saliva escapes into surrounding tissues after trauma to the duct and is walled off by granulation or connective tissue. Alternatively, they can occur with obstruction of the salivary gland duct itself.

Despite the frequency of mucoceles, if they do not spontaneously resolve within 2-3 weeks, it is recommended that patients be referred to an oral and maxillofacial surgeon for assessment, as mucoceles can occasionally be clinically difficult to distinguish from a minor salivary gland tumour. Any mucocele should be excised and sent for pathological examination.

A ranula is a mucocele of the sublingual gland (Figure 2). The term 'ranula' is derived from its close appearance to a 'frog's belly'. Treatment involves removal of the sublingual gland as marsupialisation or incision and drainage alone leads to unacceptably high recurrence rates. ${ }^{2}$

\section{Fibroepithelial polyp}

A fibroepithelial polyp is, as its name suggests, a polypoid outgrowth of tissue from the mucosal surface, which consists of fibrous connective tissue covered by normal or hyperkeratotic epithelium. It is a consequence of exuberant healing after minor oral trauma, and it is most commonly found in the lower lip or buccal mucosa in response to occlusal trauma (Figure 3). Fibroepithelial polyps can also be ulcerated. Treatment involves surgical excision.

\section{Pyogenic granuloma}

A pyogenic granuloma appears as a raised red polypoid lesion that easily bleeds and is caused by an exaggerated 


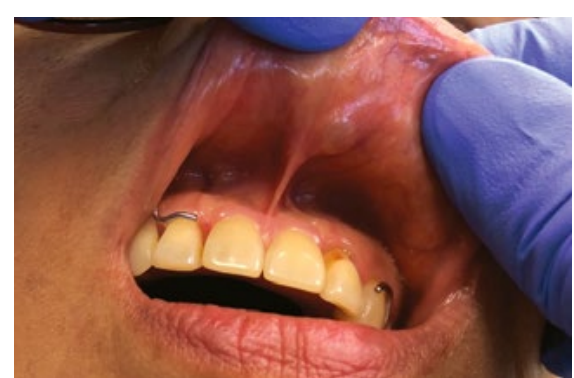

Figure 1. Mucocoele of the upper lip with domeshaped swelling and bluish tinge of saliva visible underneath the normal-appearing mucosa

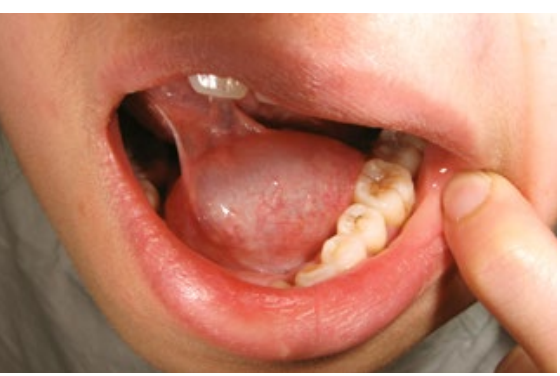

Figure 2. A ranula - a mucocele of the sublingual gland

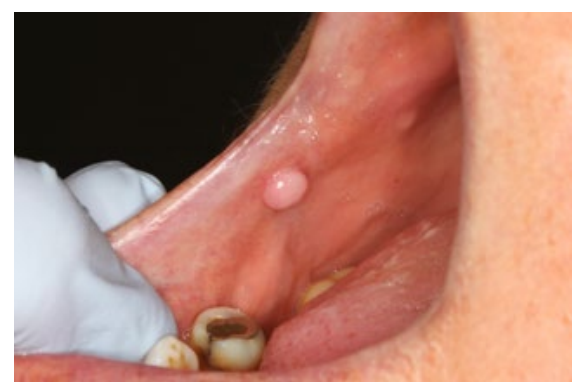

Figure 3. Fibroepithelial polyp on the right post-commissural region of the buccal mucosa with normal overlying mucosa

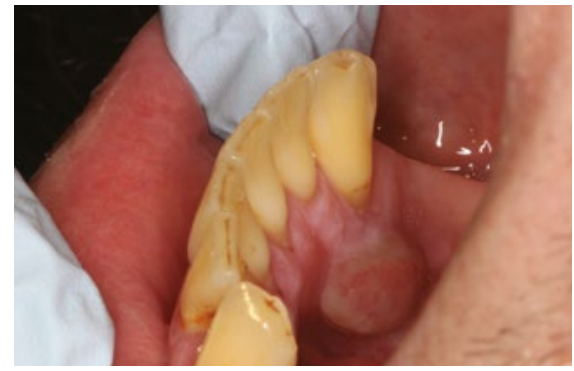

Figure 4. Pyogenic granuloma arising from the attached lingual gingiva of the lower right canine connective tissue response to minor trauma. It is most commonly found on the attached gingiva, followed by the lateral aspect of the tongue, lower lip and buccal mucosa (Figure 4 ). When found in pregnant women, pyogenic granulomas are referred to as a 'pregnancy epulis'. Treatment includes surgical excision and removal of the traumatic irritant (eg subgingival plaque).

\section{Palate}

In this article, the causes of a palatal swelling have been separated from other areas in the oral cavity because of the slightly different diagnostic considerations. Mucoceles, fibroepithelial polyps and pyogenic granulomas are rare in the hard palate because the firm, keratinised palatal mucosa is relatively resistant to trauma. The most common palatal 'swelling' is a palatal torus (discussed in the following section), but other causes of a palatal swelling are a palatal abscess or cyst (related to a non-vital upper first or second molar), minor salivary gland tumour (Figure 5), maxillary sinus tumour or lymphoma. As a result of these diagnostic possibilities, referral for further assessment is recommended for any patient with a palatal swelling.

The maxillofacial surgeon will often undertake imaging with computed tomography (CT) and/or magnetic resonance imaging prior to biopsy of a suspected minor salivary gland or maxillary sinus tumour to determine the presence and extent of underlying bone erosion; sinus, orbit or retromaxillary extension; and any radiological evidence of regional lymphadenopathy (Figure 6).

\section{The jaw 'swelling'}

\section{Exostoses and tori}

Exostoses and tori present as hard bony protuberances covered by normalappearing mucosa, and they consist of outgrowths of normal mature bone. Palatal tori are present in up to $20 \%$ of individuals and are a nodular mass of bone in the midline of the palate (Figure 7). They are asymptomatic (unless the overlying mucosa is traumatised) but may display slow growth. An orthopantomogram (OPG) may sometimes confirm the presence of exostoses and tori, but usually only once they are of a larger size $(>1.5-2 \mathrm{~cm})$.

Mandibular tori occur on the lingual surface of mandible in the premolar area, superior to the mylohyoid ridge.

Exostoses are bony protuberances that occur on the buccal aspect of the mandibular or maxillary alveolus (Figure 8).

If there is any diagnostic doubt, or the patient is anxious regarding the 'lesion', confirmation can be obtained radiologically using a cone-beam CT scan. Surgical management is not indicated unless the torus/exostosis interferes with the placement of a removable dental prosthesis (denture) or is growing, or the overlying mucosa is recurrently ulcerated.

\section{Cysts of the jaws}

Cysts of the jaws often present as a jaw swelling and are subclassified into odontogenic cysts (arising from odontogenic epithelium), non-odontogenic cysts and pseudocysts. The majority of jaw cysts are detected incidentally when the patient undergoes an OPG as part of their regular dental assessment. There are numerous types of cysts that can affect the jaws; the authors present here the most commonly encountered: the periapical cyst, dentigerous cyst and odontogenic keratocyst.

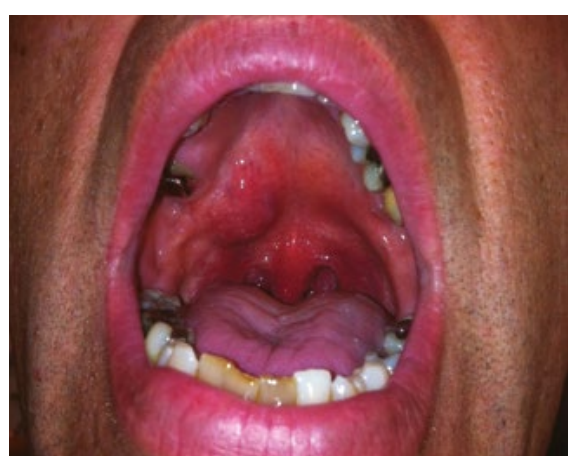

Figure 5. Right palatal swelling at the junction of the hard and soft palate. The lesion was firm on palpation. The overlying mucosa was not ulcerated. The diagnosis was mucoepidermoid carcinoma of the right palate, and treatment involved surgical excision (maxillectomy) and free flap reconstruction. 


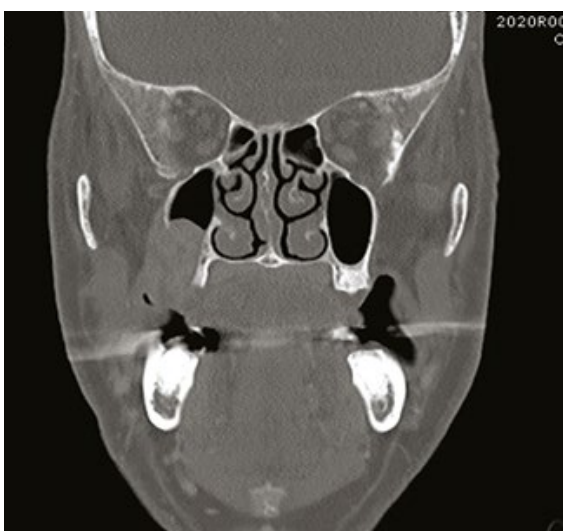

Figure 6. Coronal computed tomography scan showing extension of a right palatal tumour into the right maxillary sinus but not extending to involve the right orbital floor

\section{Periapical (radicular) cyst}

Periapical cysts are the most common cysts of the jaws and are inflammatory cysts that develop at the apex of a non-vital tooth (Figure 9). A tooth may be non-vital through dental caries, previous trauma or periodontal disease. The tooth itself may not appear to be unhealthy on visual inspection (eg secondary caries in presence of a crown), and the cyst may not be palpable unless it is large.

Prior to the formation of a cyst, a non-vital tooth may present as a sinus tract extending from the non-vital tooth apex to the buccal or palatal gingiva. A periapical abscess occurs when there is an accumulation of pus at the apex of the tooth.

The patient should be referred to their dentist for management of the non-vital tooth and periapical cyst. Management options include root canal therapy and preservation of the tooth, or extraction of the non-vital tooth and enucleation of the periapical cyst or incision and drainage of the abscess (Figure 10). Failure to manage the periapical infection adequately may lead to fascial space infection requiring hospitalisation and surgical management.

\section{Dentigerous cyst}

A dentigerous cyst is an odontogenic cyst that can develop around the crown of an impacted (unerupted) tooth (Figures 11 and 12). They are most commonly seen

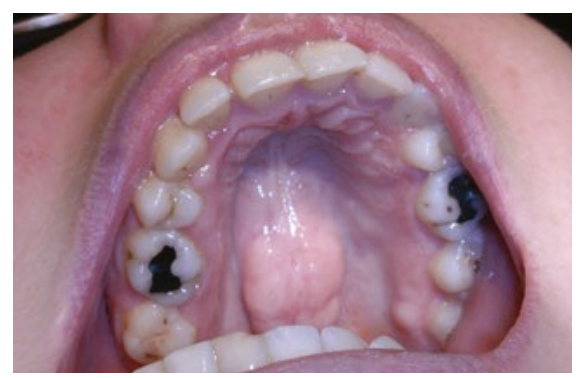

Figure 7. Torus palatinus - a bony hard protuberance found in the midline of the hard palate

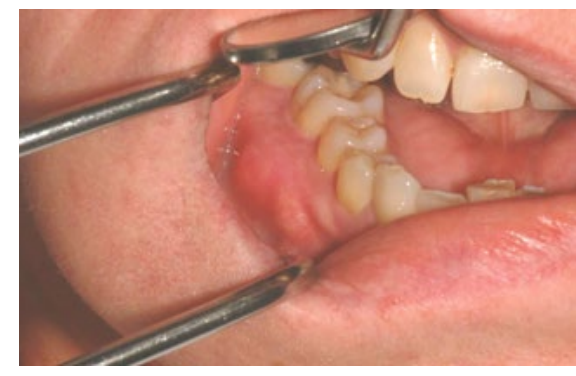

Figure 8. Bony exostoses of the lower right buccal alveolus

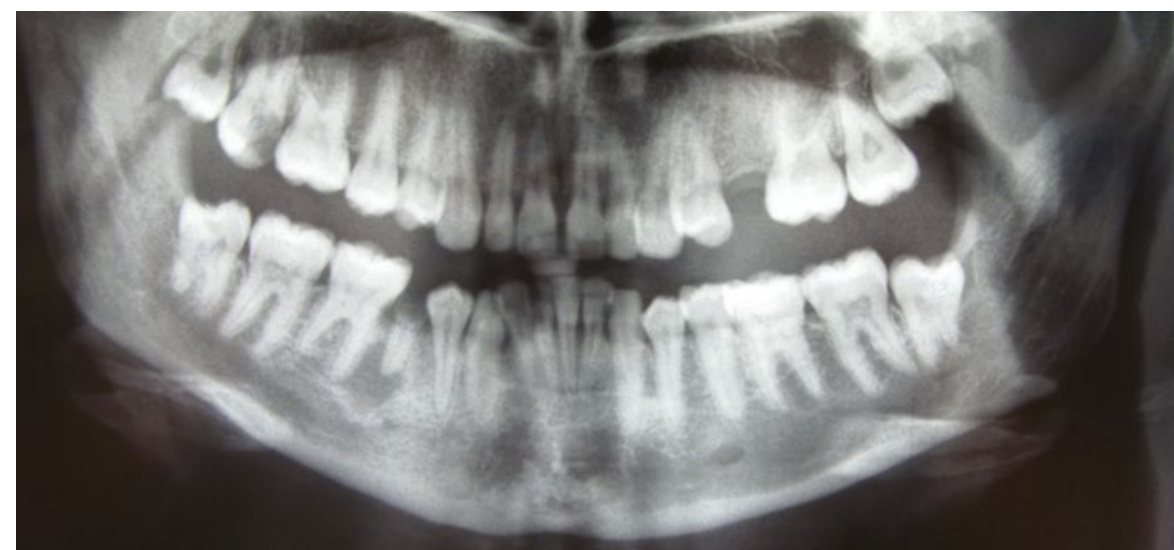

Figure 9. Orthopantomogram showing a lower right second premolar tooth with a grossly carious crown and a well-defined periapical radiolucency characteristic of a periapical (radicular) cyst arising from the non-vital tooth

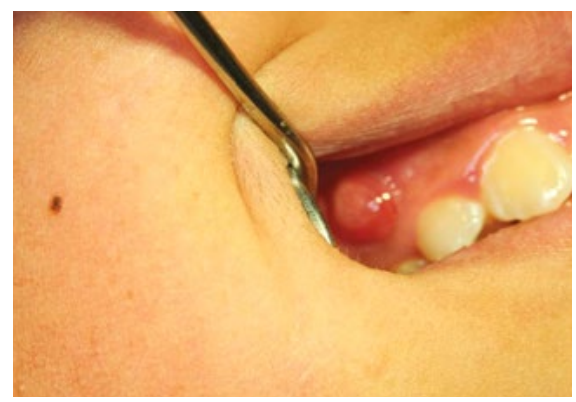

Figure 10. Draining sinus associated with a necrotic primary molar in a child aged nine years

in association with impacted third molars (wisdom teeth) or impacted canines, and they are most often diagnosed incidentally on a radiograph when investigating why an adult tooth has not erupted at the appropriate time.



Figure 11. Swelling over the mandibular alveolus with normal-appearing mucosa. The bluish tinge is the fluid visible within the cyst.

Management depends on the tooth involved and can involve enucleation of the cyst alone, with preservation of the impacted tooth, or extraction of the impacted tooth along with cyst removal. The tissue must always be 
sent for histopathological examination to determine the type of cyst as well as to exclude odontogenic tumours and malignancies of the jaws.

\section{Odontogenic keratocyst}

The odontogenic keratocyst (OKC; previously classified by the World Health Organization as a keratocystic odontogenic tumour $)^{3}$ is regarded as a cyst that can exhibit locally aggressive behaviour and has a relatively high recurrence rate after treatment. It arises from dental lamina remnants in the mandible and maxilla. In a patient with multiple OKCs, the association with naevoid basal cell carcinoma syndrome must be excluded.

OKCs occur at any age but have a peak incidence between 20-30 years and have a $2: 1$ predilection for the mandible over the maxilla. ${ }^{4}$ They may present as an asymptomatic enlargement of part of the jaw or be detected incidentally on an OPG. Radiographically they present as multilocular or unilocular radiolucencies with well-defined margins (Figures 13 and 14).

Surgical excision of the OKC is the mainstay of treatment, and various adjunctive methods have been employed to reduce the likelihood of recurrence, including peripheral ostectomy and the application of Carnoy's solution. ${ }^{5}$

\section{Fibro-osseous lesions of the jaws}

The term 'fibro-osseous lesions of the jaws' encompasses three distinct entities: fibrous dysplasia, ossifying fibroma and cemento-osseous dysplasia. They are a diverse group of lesions characterised by the replacement of normal bone with abnormal fibrous tissue that contains trabeculae of immature bone or cementum-like material.

Fibrous dysplasia is a developmental anomaly characterised by normal cancellous bone that is replaced by abnormal fibrous connective tissue in which new, non-maturing bone is formed. Onset is most common in the second and third decades of life and presents as a slow, asymptomatic enlargement of part of the mandible or maxilla. The monostotic variant affects one bone alone, whereas polyostotic fibrous dysplasia affects multiple bones.

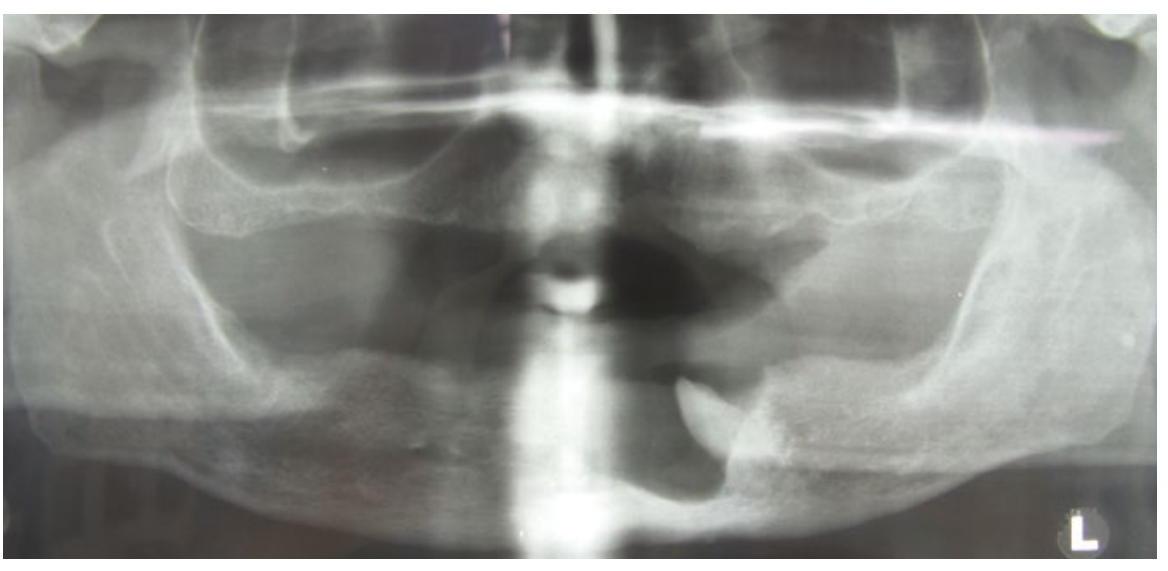

Figure 12. Orthopantomogram showing the classic appearance of a dentigerous cyst with a unilocular, well-corticated radiolucency attached to the cemento-enamel junction of the impacted lower left canine

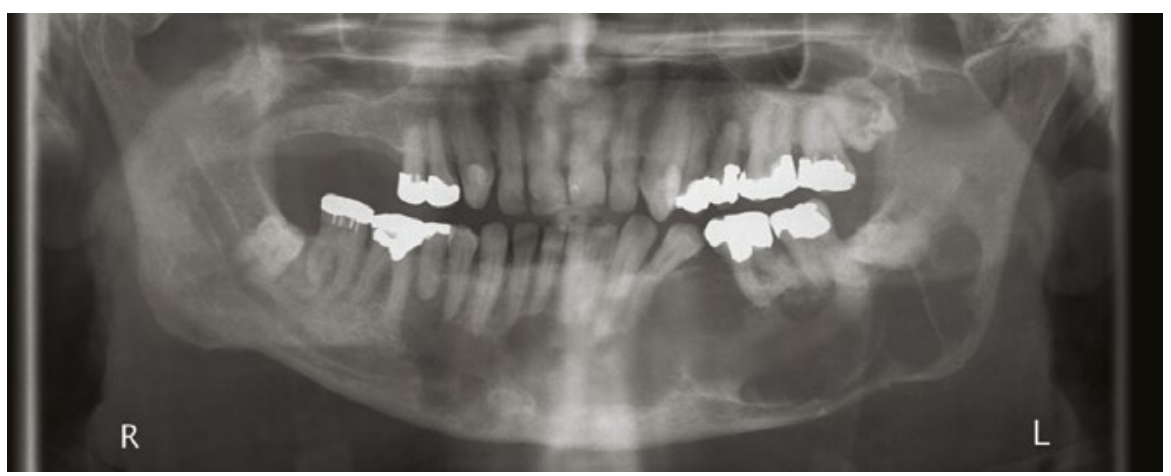

Figure 13. Orthopantomogram of an odontogenic keratocyst showing a well-corticated multilocular radiolucent lesion extending from the left ramus to the right body of the mandible, causing displacement of teeth and root resorption

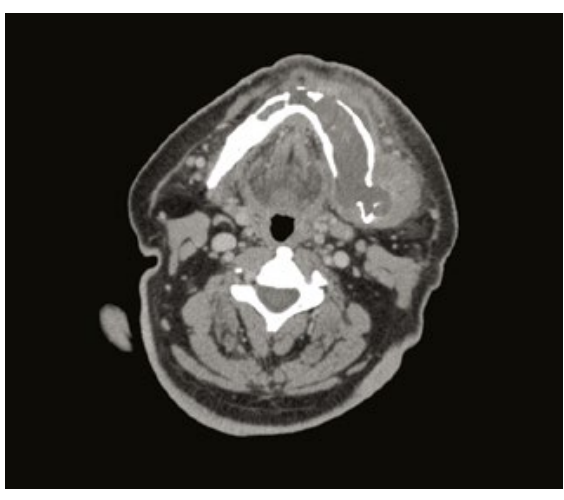

Figure 14. Axial computed tomography slice showing cortical expansion of the left mandible from the odontogenic keratocyst, with small areas of cortical breach

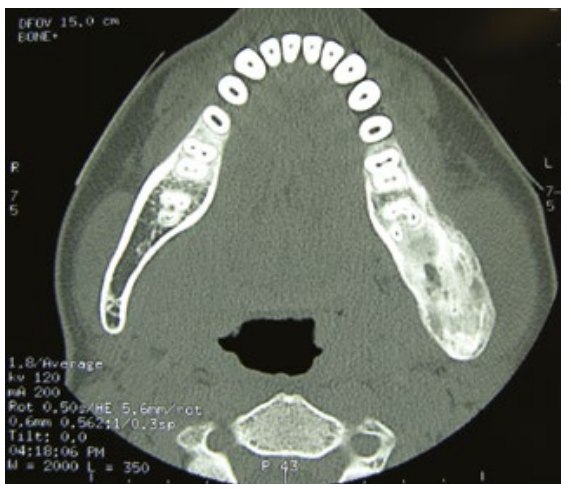

Figure 15. Axial computed tomography slice showing the mixed lucent-opaque lesion of fibrous dysplasia of the left posterior mandible (right side of the image). The patient presented after noticing several months of asymptomatic left jaw swelling. 
The radiographic appearance is variable and dependent on the stage of the condition, with the initial phase of cancellous bone being replaced by fibrous tissue appearing radiolucent. The later phase - in which new, non-maturing bone is formed within the fibrous tissue appears as a poorly defined mixed lucent/ opaque area (Figure 15).

Small areas of fibrous dysplasia may require no treatment other than biopsy confirmation and surveillance. Large lesions that cause functional or cosmetic deformity may be treated by surgical recontouring after the condition has stabilised. Medical management with bisphosphonates and monoclonal antibodies to RANKL (denosumab) has been reported in cases that are symptomatic and/or display rapid growth. ${ }^{6}$ Although uncommon, malignant transformation of fibrous dysplasia has been well described, and any sudden change in size of the lesion should alert the clinician to this possibility.

Ossifying fibroma is an uncommon lesion that presents with similar radiological characteristics to those of fibrous dysplasia, except that the margins are well defined. A more aggressive variant can be found in the paediatric population (juvenile aggressive ossifying fibroma), and the primary treatment for all forms of ossifying fibroma is surgical excision.

Cemento-osseous dysplasia generally remains confined to the apices of teeth (periapical variant) and does not commonly present as a jaw swelling. It is most commonly detected incidentally on radiographs such as periapical X-rays or OPGs, and the teeth in the region maintain their vitality.

\section{Tumours of the jaw}

Tumours of the jaw may also present as a jaw swelling and may be benign or malignant. The most common tumours of the jaw are benign odontogenic tumours such as ameloblastoma. ${ }^{8}$ Ameloblastoma can occur in all age groups but is most common in the late teenage to thirties age group.

Patients will often describe asymptomatic, slow-growing enlargement of the mandible or maxilla. The posterior

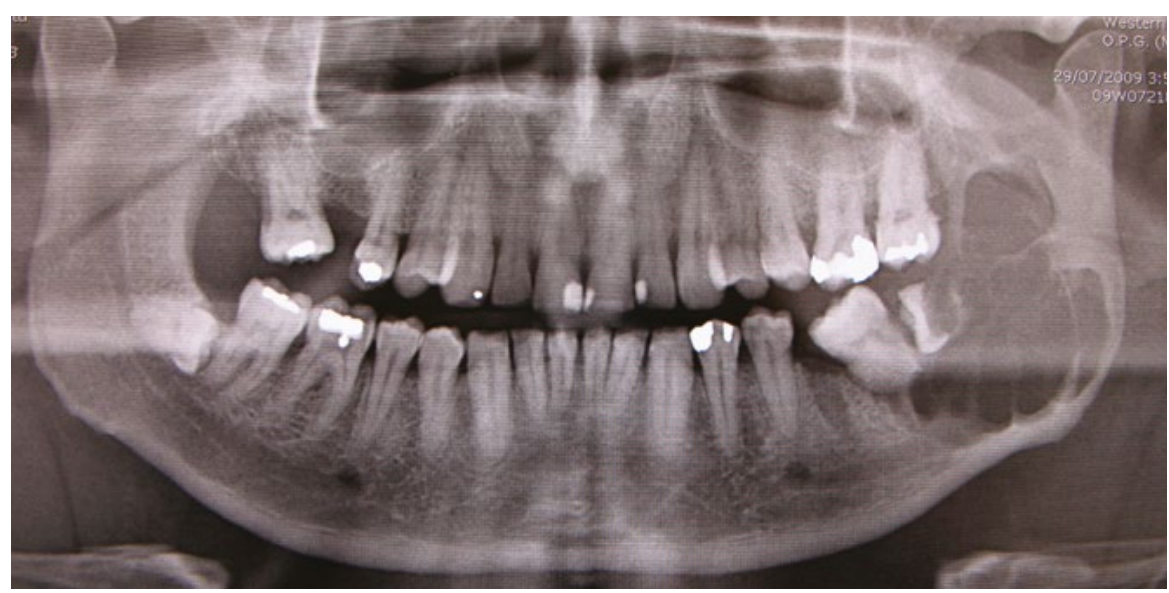

Figure 16. Orthopantomogram showing a multilocular radiolucency present in the left ramus/angle of the mandible associated with root resorption of the lower left third molar. The patient presented with left jaw swelling, and biopsy revealed solid/multicystic ameloblastoma. This required segmental resection of the mandible and free flap reconstruction.

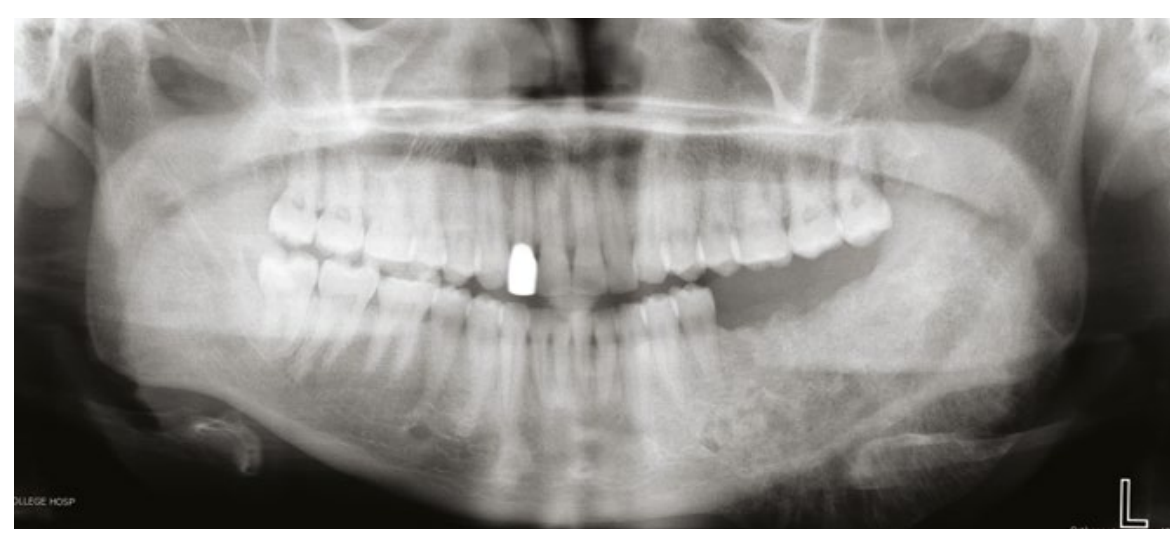

Figure 17. Orthopantomogram showing a left mandible osteosarcoma - a mixed lucent-opaque lesion extending from the lower left first premolar to involve the left ramus of the mandible with classic 'sunburst' appearance



Figure 18. Orthopantomogram post-left segmental mandibulectomy and osseocutaneous fibula free flap reconstruction for osteosarcoma of the mandible 
mandible in the third molar (wisdom tooth) region is the most common site. An OPG will show a multilocular radiolucency in the jaw that may displace or resorb teeth (Figure 16).

The treating clinician will often assess the full extent of the tumour using CT scanning and biopsy the lesion to establish the tissue diagnosis. Treatment of odontogenic tumours can vary from enucleation and curettage to excision of the lesion with a surgical margin depending on the histopathological diagnosis.

Malignant tumours of the jaw (eg osteosarcoma) are rare; however, any patient with jaw swelling should be referred to an oral and maxillofacial surgeon for further assessment and management. The radiological appearance of a malignant jaw tumour can vary significantly, from being barely visible on plain X-ray, to a radiolucent lesion or radiopaque lesion (or a mixed lucent-opaque lesion; Figure 17).

The management of malignant tumours of the jaw will almost invariably involve segmental resection of the jaw and free flap reconstruction with the possibility of adjuvant radiotherapy and chemotherapy depending on the type of tumour, pathological stage, lymph node involvement and surgical margin (Figure 18). ${ }^{9}$

\section{Conclusion}

In this article and the accompanying article, the authors have attempted to provide a brief 'Cook's tour' through the pathology of the oral cavity. There are many conditions that have been omitted because of the breadth of the topic. If readers are interested in pursuing greater depth of knowledge in oral pathology, the authors would direct them to the excellent books published by Regezi et $\mathrm{al}^{10}$ or Cawson and Odell ${ }^{11}$ on oral pathology.

\section{Key points}

- The vast majority of submucosal swellings are inflammatory lesions related to minor oral trauma; however, all submucosal swellings should be referred for excision to ensure that a minor salivary gland tumour is not overlooked.

- The differential diagnosis for a palatal swelling is slightly different from swelling in other oral cavity locations, and once a palatal torus has been excluded (midline, bony hard protuberance with normal overlying mucosa) all other palatal swellings should be referred for assessment.

- An OPG should be the first radiograph ordered in the investigation of jaw swelling as it can show jaw pathology related to teeth, odontogenic cysts and tumours, and bone pathology.

- Any tissue excised from the oral cavity should be sent for histopathological examination as the clinical or radiological appearance alone is insufficient to ensure a correct diagnosis.

\section{Authors}

Timothy Wong MBBS (Hons), BDSc (Hons), FRACDS (OMS), Oral and Maxillofacial Surgeon, Royal Melbourne Hospital, Victorian Comprehensive Cancer Centre, Vic. tscwong1@gmail.com Tami Yap BDSc (Hons), DCD, PhD, FRACDS, FOMAA, Senior Lecturer, Melbourne Dental School, The University of Melbourne, Vic; Oral Medicine Consultant, Royal Melbourne Hospital, Victorian Comprehensive Cancer Centre, Vic

David Wiesenfeld MDSc (Melb), FDSRCPS (Glas), FRACDS (OMS), Director, Head and Neck Tumour Stream, Lead in H\&N Research and Education, Victorian Comprehensive Cancer Centre, Vic; Honorary Clinical Professor, The University of Melbourne, Vic

Competing interests: None.

Funding: None.

Provenance and peer review: Commissioned, externally peer reviewed.

\section{References}

1. Wong T, Yap T, Wiesenfeld D. Common benign and malignant oral mucosal disease. Aust J Gen Pract 2020;49(9):568-73.

2. Baurmash HD. Mucoceles and ranulas. J Oral Maxillofac Surg 2003;61(3):369-78. doi: 10.1053/ joms.2003.50074.

3. Barnes L, Eveson JW, Reichart P, Sidransky D. World Health Organization classification of tumours. Pathology and genetics of head and neck tumours. Lyon, FR: IARC Press, 2005.

4. Soames JV, Southam JC. Oral pathology. 2nd edn. Oxford, UK: Oxford University Press, 1993.

5. Blanas N, Freund B, Schwartz M, Furst IM. Systematic review of the treatment and prognosis of the odontogenic keratocyst. Oral Surg Oral Med Oral Pathol Oral Radiol Endod 2000;90(5):553-58 doi: $10.1067 /$ moe.2000.110814

6. Couturier A, Aumaître O, Gilain L, Jean B, Mom T, André M. Craniofacial fibrous dysplasia: A 10-case series. Eur Ann Otorhinolaryngol Head Neck Dis 2017;134(4):229-35. doi: 10.1016/j. anorl.2017.02.004.
7. Wagner VP, Carlos R, Romañach MJ, et al. Malignant transformation of craniomaxillofacial fibro-osseous lesions: A systematic review. J Oral Pathol Med 2019;48(6):441-50. doi: 10.1111/ jop.12867.

8. Speight PM, Takata T. New tumour entities in the 4th edition of the World Health Organization classification of head and neck tumours: Odontogenic and maxillofacial bone tumours. Virchows Arch 2018;472(3):331-39. doi: 10.1007/ s00428-017-2182-3.

9. Colevas AD, Yom SS, Pfister DG, et al. NCCN guidelines insights: Head and neck cancers, version 1.2018. J Natl Compr Canc Netw 2018;16(5):479-90. doi: 10.6004/jnccn.2018.0026.

10. Regezi JA, Sciubba JJ, Jordan RCK. Oral pathology: Clinical pathologic correlations. 6th edn. St. Louis, MO: Elsevier Saunders, 2011.

11. Cawson RA, Odell EW. Cawson's essentials of oral pathology and oral medicine. 8th edn. Edinburgh, UK: Churchill Livingstone Elsevier, 2008. 